\title{
Impact of Longitudinal Plasma Leucine Levels on the Intellectual Outcome in Patients with Classic MSUD
}

\author{
BJÖRN HOFFMANN, CHRISTOPH HELBLING, PETER SCHADEWALDT, AND UDO WENDEL \\ Department of General Pediatrics [B.H., C.H., P.S., U.W.], Heinrich-Heine-University, Duesseldorf, D-40225 Germany; \\ Children's Hospital [C.H.], University of Bonn, D-53127 Bonn, Germany
}

\begin{abstract}
Maple syrup urine disease (MSUD) is an inherited deficiency of branched chain $\alpha$-ketoacid dehydrogenase (BCKDH) activity impairing the degradation of the branched chain amino acids valine, leucine, and isoleucine. Classic MSUD may lead to severe neonatal encephalopathy including coma and impaired cognitive outcome in later life. Early start of dietary treatment and careful metabolic control may improve the outcome of patients with classic MSUD. The aim of this study was to investigate the impact of long-term metabolic control assessed by plasma leucine levels on cognitive outcome in patients with classic MSUD. Plasma leucine levels of 24 patients were obtained retrospectively for the first 6 y of life and yearly medians of mean plasma leucine levels were calculated. At the age of $6 \mathrm{y}$, IQ tests were performed. Yearly medians of mean plasma leucine levels yielded three homogeneous clusters (low, intermediate, high). Patients of the low cluster showed statistically significant higher IQ scores compared with those of those of intermediate and high clusters. Long-term plasma leucine levels are associated with impaired cognitive outcome in patients with classic MSUD. To achieve the best possible intellectual outcome for affected individuals, we recommend that in infants and preschool children the target range for plasma leucine should not exceed $200 \mu \mathrm{mol} / \mathrm{L}$. (Pediatr Res 59: 17-20, 2006)
\end{abstract}

$\mathrm{M}^{\mathrm{s}}$ SUD (OMIM 248600) is an autosomal recessive inherited disorder affecting the metabolism of the branchedchain amino acids (BCAAs) valine, leucine, and isoleucine. The estimated incidence of this panethnic disorder is around 1:250 000 in Germany (1) but may be much higher in populations with a high rate of consanguineous marriages (2) or low gene shifting (3). In MSUD, the BCKDH complex is impaired and high concentrations of branched-chain amino and the corresponding 2-keto acids accumulate in patients on an unrestricted diet or during catabolic episodes. Leucine and its transamination product 2-keto isocaproic acid are thought to have potential neurotoxic effects leading to acute and chronic brain dysfunction (4), and evidence of cellular toxicity has been obtained from a neuronal cell model with decreased BCKDH activity (5).

Clinicians distinguish between the most severe classic form of MSUD with onset of encephalopathy in the first week of life and milder variant forms with later onset or even absence of cerebral symptoms. Newborns with classic MSUD are

Received April 14, 2005; accepted July 5, 2005.

Correspondence: Udo Wendel, M.D., Department of General Pediatrics, University Children's Hospital, Heinrich-Heine-University Duesseldorf, Moorenstr. 5, D-40225 Düsseldorf, Germany; e-mail: wendelu@uni-duesseldorf.de asymptomatic at birth but may develop lethargy and poor feeding within days. Untreated patients fall into coma within the first $10 \mathrm{~d}$ of life.

Newborn screening for MSUD by tandem MS technology has been started in various countries in 2000. Until then, infants with classic MSUD had most frequently reached a critical comatose state when the disorder was suspected. Diagnosis usually was made beyond $10 \mathrm{~d}$ of life due to highly increased plasma concentrations of the BCAAs. Patients then required rapid removal of the toxic substances by invasive measures, e.g. hemofiltration $(6,7)$. Once the infants have passed this critical condition they need a permanent dietary reduction of BCAA intake to prevent re-intoxication. Adequacy of dietary BCAA intake is assessed by regular measurement of plasma BCAA levels.

Without early and effective treatment children develop severe and permanent brain damage including spasticity or they even die within the first months of life (3). It has been shown that severe neonatal manifestation of classic MSUD leads to the development of impaired cognitive function in later life. Furthermore, intellectual outcome has been found to be inversely correlated with the duration of elevated plasma leucine levels during the neonatal period (8-10). In the few MSUD patients who received an immediate postnatal treatment (subsequent children in families at risk), some developed cognitive deficits in later life despite the prevention of any neonatal illness (8). Thus, additional factors besides plasma leucine levels during the neonatal period may contribute to the development of intellectual performance in later life.

This paper aims to describe a possible influence of longitudinal plasma leucine levels in childhood on the intellectual outcome in patients with classic MSUD.

\section{METHODS}

Patients. Twenty-four patients with classic MSUD (10 females, 14 males) followed by different pediatric metabolic centers in Germany, Austria, and Switzerland were enrolled in the study. All patients had reached or already passed the age of $6 \mathrm{y}$ and all were treated with a BCAA-restricted diet.

Twenty-one patients had presented with neonatal encephalopathy and had plasma leucine levels higher than $1000 \mu \mathrm{mol} / \mathrm{L}$ for a mean duration of 15.5 $\pm 3.1 \mathrm{~d}$ (range 11-21 d). Peak plasma leucine levels ranged from 1240 to

DOI: 10.1203/01.pdr.0000190571.60385.34

Abbreviations: BCAA, branched chain amino acid; BCKDH, branchedchain 2-keto acid dehydrogenase; MSUD, maple syrup urine disease; WISC-R, Wechsler Intelligence Scale for Children-Revised 
$4505 \mu \mathrm{mol} / \mathrm{L}(2739 \pm 790)$. After the resolution of neonatal illness, none had neurologic symptoms.

Three younger siblings of affected patients with MSUD have been treated prospectively after birth. These patients had no neonatal encephalopathy at all. In one patient, plasma leucine concentration never exceeded 1000 $\mu \mathrm{mol} / \mathrm{L}$. In two patients, plasma leucine did not exceed $1150 \mu \mathrm{mol} / \mathrm{L}$ and decreased to $1000 \mu \mathrm{mol} / \mathrm{L}$ by $\mathrm{d} 4$ and $\mathrm{d} 8$, respectively.

Details of the medical history and the long-term metabolic control were taken from the patients' medical files. In particular, for every patient, the postnatal time during which plasma leucine levels remained above 1000 $\mu \mathrm{mol} / \mathrm{L}$ was noted. All available plasma concentrations of leucine, valine, and isoleucine were taken from the original laboratory reports. Only plasma leucine concentrations measured by column chromatography were accepted. If several determinations of amino acids were performed within $1 \mathrm{~d}$, only the highest value was used.

At a mean age of $6 \mathrm{y}$, IQ scores were either obtained by the nonverbal Snijders-Oomen-Test, the Kaufmann Assessment Battery for Children, or the revised Wechsler Intelligence Scale for children. Part of the IQ scores were previously published by Hilliges et al. (8). Data obtained by the Kramer test (three additional patients) were not used for statistic analysis due to a lack of comparability with the other tests. In four patients, no evaluation of cognitive capacity had been performed. However, data for these patients were used to compare long-term plasma leucine levels and for cluster analysis.

All patients gave informed consent, and the study was approved by the local ethic review board.

Calculations and statistics. More than 12,000 plasma leucine values were appraised with a mean of $32 \pm 14$ analyses per patient and year. For each patient, the yearly median values of all quantitative plasma leucine levels were calculated. The courses of the first 6 yearly medians were grouped by "hierarchic cluster analysis" using Ward's procedure. The squared Euclid distance was used as distance dimension. Results of the cluster analysis were validated using the discriminant analysis. The IQs of the different clusters were compared using the Kruskal-Wallis test. Differences showing a $p$ value $<0.05$ were regarded as statistically significant. All statistical analysis were performed using the SPSS program.

\section{RESULTS}

The patients' mean age at the time of evaluation was 13.1 ( \pm 6.5 ) y. The yearly medians of plasma leucine levels are compiled in Table 1 . The profiles of the first six yearly medians were grouped by cluster analyses into three clusters, designated as low, intermediate, and high (Fig. 1).

Patients in the low cluster $(n=8)$ showed a mean of yearly median plasma leucine level of $189 \pm 82 \mu \mathrm{mol} / \mathrm{L}$, which is approximately 1.2 -fold of the upper normal value (Table 2 ; range of normal values is $77-153 \mu \mathrm{mol} / \mathrm{L}$ ) (11).

The intermediate cluster included 13 patients with a mean of yearly medians of plasma leucine concentration of $379 \pm$ $147 \mu \mathrm{mol} / \mathrm{L}$ and thus not exceeding threefold of normal values (Table 2).

The high cluster consisted of only three patients. The mean of median plasma leucine levels was $572 \pm 217 \mu \mathrm{mol} / \mathrm{L}$, which is approximately 4.5 -fold of normal values (Table 2 ).

Interestingly, the means of the median plasma leucine levels in the high and intermediate clusters were nearly identical during the first 2 y of life. From y 3 onward, the means of the yearly median plasma leucine levels differed between the two clusters (Fig. 2). No significant differences were found for either age at the time of evaluation or for gender between the patients of the three clusters.

Cognitive outcome and relationship with metabolic control. In total, the IQ scores of 15 patients were obtained for statistic analysis (five in the low cluster, eight in the intermediate cluster, and two in the high cluster (Table 3). The patient's IQ scores were related to longitudinal plasma leucine concentrations. For the three clusters, the mean IQ scores
Table 1. Yearly medians of plasma leucine levels for 24 patients with MSUD over 6 y

\begin{tabular}{crrrrrr}
\hline & \multicolumn{5}{c}{ Yearly medians of plasma leucine } \\
& \multicolumn{5}{c}{ concentration $(\mu \mathrm{mol} / \mathrm{L})$} \\
\cline { 2 - 7 } Patient & 1 st y & 2nd y & 3rd y & 4th y & 5 th y & 6 th y \\
\hline 1 & 302 & 321 & 420 & 187 & 336 & 443 \\
2 & 290 & 328 & 298 & 298 & 382 & 412 \\
3 & 187 & 208 & 183 & 282 & 221 & 210 \\
4 & 130 & 252 & 412 & 466 & 321 & 351 \\
5 & 375 & 527 & 462 & 553 & 908 & 695 \\
6 & 275 & 481 & 573 & 626 & 733 & 546 \\
7 & 321 & 271 & 443 & 336 & 450 & 538 \\
8 & 191 & 321 & 397 & 374 & 450 & 450 \\
9 & 363 & 527 & 573 & 519 & 611 & 191 \\
10 & 183 & 198 & 332 & 384 & 377 & 426 \\
11 & 282 & 557 & 294 & 496 & 500 & 515 \\
12 & 305 & 313 & 183 & 218 & 176 & 237 \\
13 & 271 & 378 & 344 & 656 & 374 & 485 \\
14 & 208 & 593 & 58 & 98 & 379 & 755 \\
15 & 168 & 168 & 397 & 336 & 580 & 476 \\
16 & 88 & 84 & 179 & 134 & 221 & 221 \\
17 & 466 & 901 & 134 & 439 & 267 & 322 \\
18 & 92 & 115 & 176 & 103 & 92 & 172 \\
19 & 313 & 252 & 263 & 282 & 114 & 142 \\
20 & 256 & 105 & 116 & 322 & 266 & 195 \\
21 & 340 & 202 & 343 & 80 & 98 & 250 \\
22 & 563 & 298 & 359 & 466 & 412 & 401 \\
23 & 115 & 74 & 68 & 79 & 99 & 291 \\
24 & 244 & 153 & 763 & 649 & 862 & 863 \\
\hline
\end{tabular}

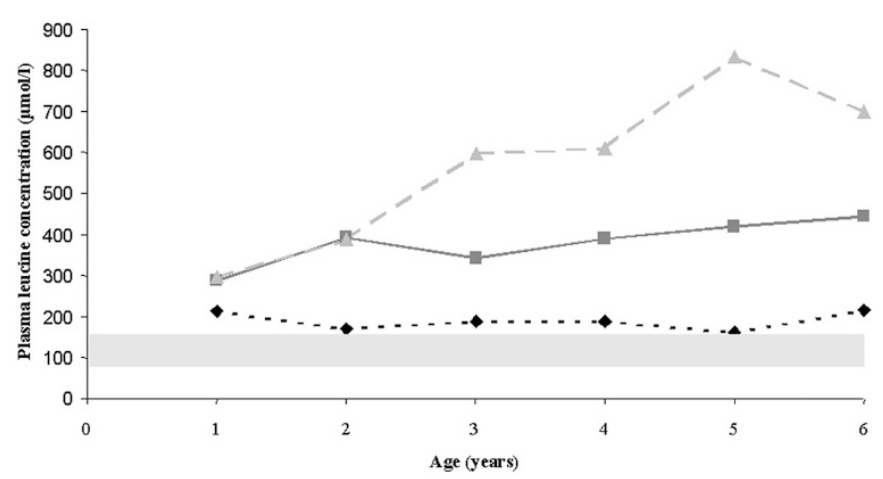

Figure 1. Plasma leucine levels in 25 patients with classic MSUD over $6 \mathrm{y}$. Means of yearly median plasma leucine levels $(\mu \mathrm{mol} / \mathrm{L})$ for 24 patients with MSUD according to the three clusters. The low cluster $(\bullet)$ comprises eight patients, the intermediate cluster (ם) 13 patients, and the high cluster ( $\mathbf{\Delta})$ three patients, respectively. Shading shows normal range of plasma leucine concentration.

showed an inverse relationship to the average plasma leucine levels and thus to the quality of metabolic control.

Median IQ score of patients in the low cluster was 102 (range, 86-108). In the intermediate cluster, it was 76 (range, 57-99), and in the high cluster, it was 57 (range, 50-64). The differences of the IQ scores between the three clusters were statistically significant $(p<0.05$; Fig. 2).

\section{DISCUSSION}

This study provides evidence that the long-term metabolic control of patients with the classic form of MSUD has a major impact on the patient's cognitive outcome. 
Table 2. Means ( $\pm S D$ ) of yearly median plasma leucine levels over 6 y according to the three clusters

Means ( $\pm \mathrm{SD})$ of yearly median plasma leucine levels $(\mu \mathrm{mol} / \mathrm{L})$

\begin{tabular}{llllrrr}
\cline { 2 - 6 } Cluster & \multicolumn{1}{c}{ 1st y } & 2nd y & 3rd y & 4th y & 5th y \\
\hline Low & $212( \pm 105)$ & $169( \pm 87)$ & $189( \pm 84)$ & $188( \pm 100)$ & $161( \pm 69)$ & $215( \pm 46)$ \\
Intermediate & $288( \pm 123)$ & $393( \pm 201)$ & $343( \pm 132)$ & $389.0( \pm 145)$ & $418( \pm 99)$ & $443.0( \pm 130)$ \\
High & $298( \pm 68)$ & $387( \pm 204)$ & $599( \pm 152)$ & $609( \pm 50)$ & $834( \pm 91)$ & $701( \pm 159)$ \\
\hline
\end{tabular}

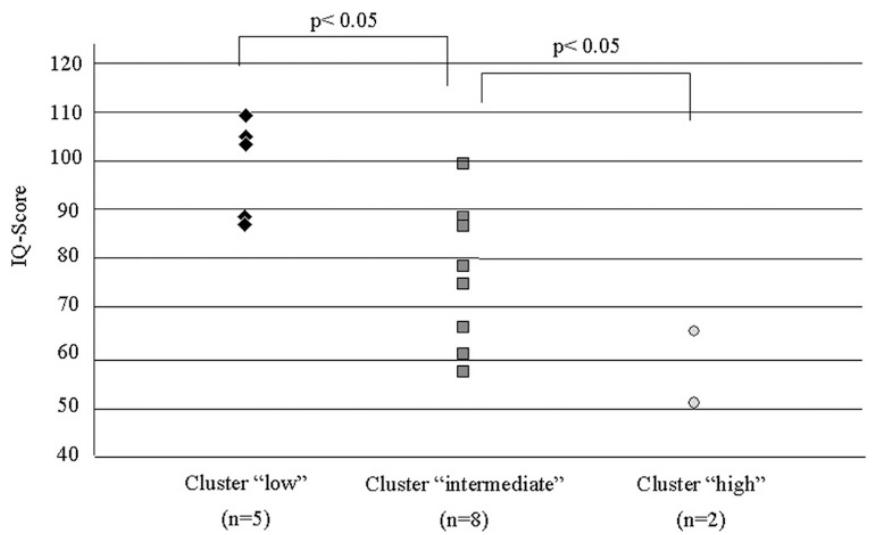

Figure 2. IQ scores of patients with MSUD in the three clusters $(n=15)$. Patients with best metabolic long-term control showed best cognitive capacity. Patients with poorer metabolic long-term control scored significantly lower on IQ.

Irrespective of neonatal encephalopathy, normal cognitive outcome is achievable if long-term plasma leucine levels are close to normal. However, in patients without neonatal encephalopathy and thus with optimal starting-point conditions, intellectual outcome seems to be heavily influenced by longterm plasma leucine levels.

The cohort of patients with MSUD described here is relatively large compared with the incidence of the disease and the number of patients attending each single metabolic centre. Nevertheless, the potential of statistical examinations is limited by the overall small number of 24 patients. Despite these limitations we tried to identify influencing and/or predicting factors for the outcome of MSUD.

All patients were born, diagnosed, and treated in Austria, Switzerland, or Germany. However, their parents were immigrants in $62.5 \%$, whereas in $37.5 \%$, the parents were from the native population. Children from immigrant families are clearly underrepresented in the low cluster (37.5\%) and are more frequent in the intermediate and high clusters. Possible explanations for that may be distinct cultural and health beliefs as well as linguistic deficiencies in the migrant community. Therefore, proper communication on the basics, characteristics, and treatment measures of that disease between the therapeutic team and the patient's family is essential. The use of written instructions in the patient's and his or her parents' primary language may be helpful in maintaining better outcome. Staff with adequate language skills and knowledge of cultural understanding is also preferred.

Despite the fact that all patients received treatment in German-speaking countries, there may be differences in the treatment recommendation among the individual metabolic centers regarding the long-term plasma leucine levels that
Table 3. Plasma leucine levels and IQ performance in the three clusters of patients with MSUD

\begin{tabular}{|c|c|c|c|c|}
\hline Cluster & $\begin{array}{l}\text { Peak plasma } \\
\text { leucine } \\
\text { concentration } \\
(\mu \mathrm{mol} / \mathrm{L})\end{array}$ & $\begin{array}{c}\text { Duration of } \\
\text { plasma leucine } \\
>1000 \mu \mathrm{mol} / \mathrm{L} \\
\text { (d) }\end{array}$ & IQ & $\begin{array}{l}\text { Age at IQ } \\
\text { testing (y) }\end{array}$ \\
\hline \multirow[t]{8}{*}{ I } & 3411 & 13 & $*$ & $*$ \\
\hline & 4505 & 17 & 102 & 4 \\
\hline & [1145] & [4] & $\dagger$ & $\dagger$ \\
\hline & 3450 & 14 & 108 & 7 \\
\hline & 2824 & 13 & 104 & 7 \\
\hline & 2336 & 15 & $\dagger$ & $\dagger$ \\
\hline & 2733 & 14 & 87 & 7 \\
\hline & 1700 & 14 & 86 & 7 \\
\hline \multirow[t]{13}{*}{ II } & [1122] & [8] & [57] & [13] \\
\hline & 2573 & 21 & 60 & 13 \\
\hline & 2480 & 12 & 99 & 8 \\
\hline & 3618 & 19 & 57 & 6 \\
\hline & 1496 & 16 & 77 & 6 \\
\hline & 3794 & 11 & 65 & 6 \\
\hline & 1481 & 11 & $*$ & $*$ \\
\hline & 3344 & 16 & 87 & 4 \\
\hline & 2878 & 11 & $\dagger$ & $\dagger$ \\
\hline & 1240 & 21 & $*$ & $*$ \\
\hline & 2644 & 18 & 86 & 10 \\
\hline & 2560 & 17 & 75 & 6 \\
\hline & 2744 & 20 & $* *$ & $* *$ \\
\hline \multirow[t]{3}{*}{ III } & 2745 & 16 & 64 & 8 \\
\hline & [802] & [1] & [65] & [7] \\
\hline & 2870 & 15 & $<50$ & 6 \\
\hline
\end{tabular}

* Only results from Kramer test were documented when no comparison with the other IQ test was possible.

$\dagger$ No IQ test performed.

Numbers in brackets: prospectively treated patients, IQ scores not used for statistic analysis.

should be appreciated. Hence, patients could have been assigned a priori to a specific cluster.

Interestingly, the high cluster comprised more patients who were older (not significant). Yet, it remains open whether the younger patients had more benefit from improved knowledge of the treatment of MSUD. Another explanation could be that with age and independence from parental surveillance, dietary control became worse.

Finally, children with moderate and poor long-term metabolic control had more severe metabolic decompensations than patients with excellent metabolic long-term control (data not shown). This may suggest that knowledgeable parents may recognize an imminent derangement during catabolic stress in time and start preventive dietary measures early.

Previous authors have indicated that early and meticulous treatment of patients with MSUD can result in normal intellectual outcome (9). However, Kaplan and colleagues (9) unfortunately mixed developmental quotients obtained from 
the Mental Scale of the Bayley Scale of Infant Development with "real" IQ test results from Stanford-Binet or WISC-R.

Additionally, there is a clear correlation between the duration of plasma leucine levels above $1000 \mu \mathrm{mol} / \mathrm{L}$ in the neonatal period and intellectual outcome $(8,10)$. Nevertheless, early diagnosis of MSUD and rapid lowering of plasma leucine concentrations to less than $1000 \mu \mathrm{mol} / \mathrm{L}$ in neonates are not the only crucial points for good intellectual performance in later life. The long-term biochemical control is another, and maybe the most important, factor that has an extremely important influence on the intellectual outcome in children with MSUD. Kasinski et al. (5) have identified leucine as the major cell toxin in neuronal cells with impaired BCKD activity. Araujo and coworkers (12) speculated that a decrease of essential amino acids in brain may lead to reduction of protein and neurotransmitter synthesis in MSUD. However, these observations derive from an animal model and the authors point out that the relevance for MSUD patients yet has to be evaluated. Additionally, Zielke et al. (13) investigated the competition for the transport of large neutral amino acids across the blood-brain barrier as a possible pathomechanism in MSUD. Recently, Yudkoff et al. (14) reported on a depletion of different amino acids in the brain, including glutamate, glutamine, aspartate, and alanine. The authors concluded that this depletion results in a compromise of energy metabolism and a diminished rate of protein synthesis. In summary, it becomes clear from all these reports that longterm metabolic control is essential for normal brain development and best possible neurocognitive outcome.

To enable parents and patients to come up with the best treatment results, clear and comprehensive information on treatment, including the target range for plasma leucine levels, and continuous training is essential. Furthermore, regular biochemical monitoring and early and sufficient intervention during catabolic episodes is mandatory.

The present retrospective study shows that in infants and preschool children the target range for plasma leucine should not exceed $200 \mu \mathrm{mol} / \mathrm{L}$ to achieve the best possible intellectual performance. This may not be achieved during catabolic episodes that may occur during intercurrent illnesses but should be the goal for long-term treatment.
Acknowledgments. This paper contains parts of the doctoral thesis of C.H. The authors very much appreciate the support and efforts of the following colleagues who kindly provided laboratory reports and clinical data of their patients: C. Aring, Cologne; A. Bökenkamp, Bonn; J. Kreuder, Gießen; D. Leupold, Ulm; E. Mönch, Berlin; C. Renner, Erlangen; I. Knerr, Erlangen; H.-G. Koch, Münster and Braunschweig; R. Mallmann, Bonn and Essen; E. Mayatepek, Heidelberg and Duesseldorf; H. Niederhoff, Freiburg; S. Schweitzer-Krantz, Hannover and Duesseldorf (all Germany); and B. Baumgartner, Basel, Switzerland; D. Skladal, Innsbruck; B. Plecko, Graz (all Austria). G. Kamp, Düsseldorf, Germany, kindly gave advice on the analysis of cognitive testings.

\section{REFERENCES}

1. Schulze A, Lindner M, Kohlmuller D, Olgemoller K, Mayatepek E, Hoffmann GF 2003 Expanded newborn screening for inborn errors of metabolism by electrospray ionization-tandem mass spectrometry: results, outcome, and implications. Pediatrics 111:1399-1406

2. Teebi AS 1994 Autosomal recessive disorders among Arabs: an overview from Kuwait. J Med Genet 31:224-233

3. Morton DH, Strauss KA, Robinson DL, Puffenberger EG, Kelley RI 2002 Diagnosis and treatment of maple syrup disease: a study of 36 patients. Pediatrics 109:9991008

4. Chuang D, Shih V 2001 Maple syrup urine disease (branched-chain ketoaciduria). In: Scriver C, Beaudet A, Sly W, Valle D (eds) The Metabolic \& Molecular Bases of Inherited Disease. McGraw-Hill, New York, pp 1971-2005

5. Kasinski A, Doering CB, Danner DJ 2004 Leucine toxicity in a neuronal cell model with inhibited branched chain amino acid catabolism. Brain Res Mol Brain Res 122:180-187

6. Jouvet P, Poggi F, Rabier D, Michel JL, Hubert P, Sposito M, Saudubray JM, Man NK 1997 Continuous venovenous haemodiafiltration in the acute phase of neonatal maple syrup urine disease. J Inherit Metab Dis 20:463-472

7. Schaefer F, Straube E, Oh J, Mehls O, Mayatepek E 1999 Dialysis in neonates with inborn errors of metabolism. Nephrol Dial Transplant 14:910-918

8. Hilliges C, Awiszus D, Wendel U 1993 Intellectual performance of children with maple syrup urine disease. Eur J Pediatr 152:144-147

9. Kaplan P, Mazur A, Field M, Berlin JA, Berry GT, Heidenreich R, Yudkoff M, Segal S 1991 Intellectual outcome in children with maple syrup urine disease. J Pediatr 119:46-50

10. Snyderman SE 1988 Treatment outcome of maple syrup urine disease. Acta Paediatr Jpn 30:417-424

11. Lepage N, McDonald N, Dallaire L, Lambert M 1997 Age-specific distribution of plasma amino acid concentrations in a healthy pediatric population. Clin Chem 43:2397-2402

12. Araujo P, Wassermann GF, Tallini K, Furlanetto V, Vargas CR, Wannmacher CM, Dutra-Filho CS, Wyse AT, Wajner M 2001 Reduction of large neutral amino acid levels in plasma and brain of hyperleucinemic rats. Neurochem Int 38:529-537

13. Zielke HR, Zielke CL, Baab PJ, Collins RM 2002 Large neutral amino acids autoexchange when infused by microdialysis into the rat brain: implication for maple syrup urine disease and phenylketonia. Neurochem Int 40: 347-354

14. Yudkoff M, Daikhin Y, Nissim I, Horyn O, Luhovyy B, Lazarow A, Nissim I 2005 Brain amino acid requirements and toxicity: the example of leucine. J Nutr 135: 1531S-1538S 